lage: a nineteenth century riverine community in southwestern Alaska, 1968; Akulivikchuk: a nineteenth century Eskimo village on the Nushagak River, Alaska, 1970; Nushagak: an historic trading center in southwestern Alaska, 1972) for specific details on individual villages.

The nineteenth century in Alaska has been badly neglected by archaeologists, and southwestern riverine Eskimos have been particularly slighted. Alone, the monograph reviewed is not a particularly impressive tome; however, together with the others in the series, they provide one of the first extensive and intensive ethnohistorical studies of a southwestern Alaska Eskimo population and, in this, the series is a major contribution to our understanding of a period of very rapid culture contact and change.

JOAN B. TOWNSEND

University of Manitoba

Winnipeg, Canada

\title{
Reactions to Reviews
}

Review by Olive R. Jones in "Historical Archaeology 1972." pp., 102-108, of Glass from Fort Michilimackinac: AClassification System for Eighteenth Century Glass.

Miss Jones has made some helpful critical remarks concerning my glass report (Glass from Fort Michilimackinac) but others of her criticisms arise, I feel, from a lack of understanding of the principles in the classification.

Classification organizes items by means of certain of their shared and discrete attributes. The classification used here is a form of a key diagram. This does not work like a code, Class I, Type 2, Variety "a" does not have any intrinsic meaning; it is necessary in all classifications to know what Class, type, and variety in this classification refers to.

There are two separate classifications, one for the whole and one for the fragmentary items, because the same criteria cannot apply to both; a neck fragment does not possess all or a majority of the attributes which are possessed by the whole vessel. It is true that a straight blue-green neck probably belongs to the square-sectioned blue-green bottle, but this bottle occurs in two sizes and, for all I know, several other styles as well which I have not seen. The congruence between the two classifications was pointed out, but one would not need to look for an individual "artifact" in both places, as if the item were whole it would be found in one grouping and if fragmentary in another. I haven't the faintest idea what the third place to look for the artifact is that she mentions, unless she refers to the photos, the placement of which was the choice of the editor.

It should be understood that a formal classification is not forced on the material, it is derived out of the material, its interrelationships and shared and discrete attributes. Descriptive attributes do not have equal value in a classification. To divide bottles, for example, by string rims at a high level would cause an excessive amount of branching. Several different arrangements of the attributes were considered and the present was the most economical.

Miss Jones has made some helpful suggestions and since I do not consider the classification an end but a beginning, hopefully some can be incorporated at a later date. I agree with her that bases could be included; at the time I did my work I did not have her excellent 1971 study on these (Olive Jones, "Glass Bottle Push-ups and Pontil Marks," Historical Archaeology 1971:62-73), and was not aware of the significance of all the variation.

MARGARET K. BROWN Evanston, Illinois 